\title{
Estudio exploratorio de satisfacción laboral de los profesionales de enfermería generalistas y especialistas (matrona)
}

\author{
Exploratory study on the general and specialist nursing \\ professionals (midwife) laboral satisfaction
}

\section{Estudo exploratório da satisfação no trabalho dos enfermeiros generalistas e especialistas em obstetrícia e ginecologia (parteiras)}

César Carrillo García ${ }^{1}$; María Isabel Ríos Rísquez²; María de los Ángeles Castaño Molina ${ }^{3}$; María Dolores

NicolásVigueras"; José Arnau Sánchez ${ }^{5}$, M.E. Martínez Roche ${ }^{6}$

${ }^{1}$ Doctor en Enfermería. Profesor Asociado Clínico Ciencias de la Salud. Facultad de Enfermería. Universidad de Murcia. Enfermero Hospital Universitario José María Morales Meseguer.

${ }^{2}$ Doctora en Psicología. Profesora Asociada Clínica Ciencias de la Salud. Facultad de Enfermería. Universidad de Murcia. Enfermera Hospital Universitario José María Morales Meseguer.

${ }^{3}$ Doctora en Filosofía. Profesora Asociada Académica. Facultad de Enfermería.

Universidad de Murcia. Matrona Hospital Comarcal del Noroeste.

${ }^{4}$ Grado en Enfermería. Matrona Hospital Universitario Santa Lucia.

${ }^{5}$ Doctor en Enfermería. Matrón Centro de Salud de Vistabella.

${ }^{6}$ Profesora Titular de Universidad. Facultad de Enfermería. Universidad de Murcia. (Investigadora Principal).

Cómo citar este artículo en edición digital: Carrillo García, C; Ríos Rísquez, $M^{a}$.I; Castaño Molina, Ma ${ }^{a}$. ; Nicolás Vigueras, Ma .D; Arnau Sánchez, J. y Martínez Roche, Ma.E. (2014) Estudio exploratorio de satisfacción laboral de los profesionales de enfermería generalistas y especialistas (matrona). Cultura de los Cuidados (Edición digital) 18, 39.

Disponible en: http://dx.doi.org/10.7184/cuid.2014.39.12>

Correspondencia: César Carrillo García. Campus Universitario de Espinardo. Edificio 23. Departamento de Enfermería. Universidad de Murcia. 30100. Espinardo. Murcia.

Correo electrónico: cesarcarrillo@um.es

Recibido: 11/03/2014; Aceptado: 16/06/2014

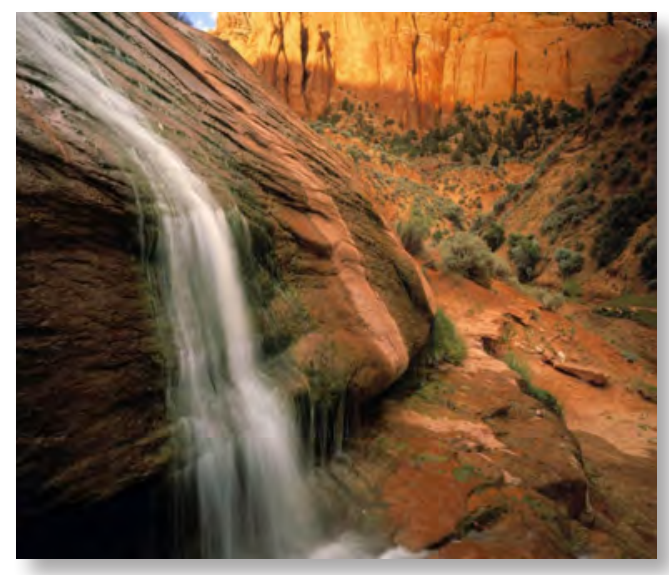

\begin{abstract}
Starting from the importance of the laboral satisfaction and its repercussion in the development of the Health Professional activity as well as its impact in the quality of the services rendered.

The aim of this study is to know, analyze and describe the expectations, needs, values and perceptions of the general and specialist nursing professionals (midwife) of three University Hospitals in relation to the satisfaction
\end{abstract}

Financiación: Proyecto de Investigación en Salud financiado por el Instituto de la Salud Carlos III. Fundación para la Formación e Investigación Sanitarias de la Región de Murcia. PI12/01776. Estudio exploratorio sobre la satisfacción de la salud laboral de los profesionales de enfermería generalistas y especialistas (matrona) de las Áreas Sanitarias I, VI y VII de la Región de Murcia. Agradecimientos: A la cofinanciación de la Unión Europea por el Fondo Europeo de Desarrollo Regional. "Una manera de hacer Europa". 
of safety at work as an influential factor in their life quality.

The design is considered from a qualitative perspective under the theoretical and methodological guidance of the social anthropology, using a theoretical sampling until reach the data saturation. The discussion groups with general and specialist nursing professionals will be used as data collection techniques.

The results of a quantitative study on the working life satisfaction at an University Hospital and the different aspects which produce the same in professionals, raise the issue of the start of the study.

As a conclusion, this kind of study will allow us to investigate reasons for the satisfaction or dissatisfaction of the nursing professionals, inasmuch as the quality of their laboral health affects directly the quality of the services rendered.

Keywords: Nursing Staff Hospital, Obstetrical Nursing, Job Satisfaction, Qualitative Research.

\section{RESUMO}

Assumimos a importância da satisfação no trabalho e o seu impacto sobre o desenvolvimento da actividade dos Profissionais da Saúde, bem como seu impacto sobre a qualidade dos serviços prestados.

O objetivo é compreender, analisar e descrever as expectativas, necessidades, valores e percepções dos Profissionais de Enfermagem generalistas e especialistas (parteiras) de três Hospitais Universitários em relação a satisfação da saúde ocupacional como um fator que influencia a sua qualidade de vida.

O designsurge a partir de uma abordagem qualitativa, sob a orientação teórico-metodológica da Antropologia Social, utilizando uma amostragem teórica até a saturação de dados.
Serám usados como técnicas de recolha de dados qualitativos os Grupos de Discusso com Profissionais de Enfermagem generalistas e especialistas.

Os resultados de um estudo quantitativo sobre a satisfação da vida de trabalho em um Hospital Universitário e os vários aspectos que produze a mesma nos profissionais, faz-nos pensar sobre o início deste estudo.

Em conclusão, um estudo desta natureza, nós permitiria investigar os porquês da satisfação e insatisfação dos Profissionais de Enfermagem, uma vez que a qualidade da saúde ocupacional deles tem uma influência directa sobre a qualidade do serviço prestado.

Palavras-chave: Recursos Humanos de Enfermagem no Hospital, Enfermagen Obstétrica, Satisfação no Emprego, Pesquisa Qualitativa.

\section{RESUMEN}

Partimos de la importancia de la satisfacción laboral y su repercusión en el desarrollo de la actividad de los Profesionales Sanitarios así como su impacto en la calidad de los servicios que prestan.

El objetivo es conocer, analizar y describir las expectativas, necesidades, valores y percepciones de los profesionales de enfermería generalistas y especialistas (matrona) de tres Hospitales Universitarios en relación a la satisfacción de la salud laboral como factor influyente en su calidad de vida.

El diseño se plantea desde un enfoque cualitativo bajo la orientación teórico-metodológica de la antropología social, utilizando un muestreo teórico hasta alcanzar la saturación de los datos. Se usarán como técnicas de recogida de datos cualitativos los grupos de discusión con profesionales de enfermería generalistas y especialistas. 
Los resultados de un estudio cuantitativo sobre la satisfacción de la vida laboral en un Hospital Universitario y los distintos aspectos que producen la misma en los profesionales, nos hacen plantearnos el inicio de este estudio.

Como conclusión, un estudio de estas características, nos permitiría indagar en los porqués de la satisfacción e insatisfacción de los profesionales de enfermería, ya que la calidad de salud laboral de los mismos influye directamente en la calidad del servicio prestado.

Palabras clave: Personal de Enfermería en Hospital, Enfermería Obstétrica, Satisfacción en el Trabajo, Investigación Cualitativa.

\section{INTRODUCCIÓN}

Para poder hablar de satisfacción laboral, se ha de definir en primer lugar el trabajo como un conjunto de actividades humanas, retribuidas o no, de carácter productivo y creativo, que mediante el uso de técnicas, instrumentos, materias o informaciones disponibles, permite obtener, producir o prestar ciertos bienes, productos o servicios. En dicha actividad la persona aporta energías, habilidades, conocimientos y otros diversos recursos y obtiene algún tipo de compensación material, psicológica y/o social (Peiró, 1986).

Cuando se habla de satisfacción laboral, ésta se define como una actitud compuesta por elementos afectivos, cognitivos y conductuales, que pueden variar en consistencia y magnitud, pueden ser obtenidos de diferentes fuentes y cumplen distintas funciones para el individuo (Sáez, 1999). Un segundo aspecto relevante a tener en cuenta en relación con el trabajo y la satisfacción laboral de los profesionales es su motivación. Ésta se halla presente en cualquier contexto de la existencia del hombre, interviniendo como mecanismo para lograr una serie de propósitos, y como impulsora de la conducta humana, siendo una pieza clave a la hora de entender porqué unas personas rinden más que otras en el desarrollo de su actividad laboral (Sáez, 1999).

Otro aspecto a tener en cuenta, es la definición de salud laboral, que según la Organización Mundial de la Salud, tiene como objetivo fomentar y mantener el más alto nivel de bienestar físico, mental y social de los trabajadores en todas las profesiones, prevenir todos los daños a la salud de éstos por las condiciones de su trabajo, protegerlos en su trabajo contra los riesgos para la salud y colocar y mantener al trabajador en un empleo que convenga a sus aptitudes psicológicas y fisiológicas (Organización Mundial de la Salud, 2013).

En cuanto al concepto de calidad de vida laboral, tiene que ver con la satisfacción, la salud y el bienestar del trabajador, y también con lo relacionado con su entorno laboral. Es una definición que pretende reconciliar aspectos del trabajo que tienen que ver con las experiencias individuales y con los objetivos organizacionales. Es, por tanto, un concepto multidimensional, formado por indicadores objetivos y subjetivos que tienen en cuenta tanto al individuo como el contexto en el que se desenvuelve (Flores, Jenaro, González-Gil, Robaina y Martín, 2010).

Numerosos autores, han señalado en sus estudios la importancia de las investigaciones en satisfacción de la vida laboral y, sobre todo en los servicios de salud, destacando la relevancia de la satisfacción laboral en los profesionales sanitarios, resaltando también las consecuencias que se derivan de esa satisfacción laboral, como son la mejora de la calidad asistencial de los servicios prestados y una mayor satisfacción de los usuarios (García de Diego, Ranchal, Biedma, Velázquez y Serrano 
del Rosal, 2008; Peiró, Silla, Sanz, Rodríguez y García, 2004; Robles, Dierssen, Martínez, Herrera, Díaz y Llorca, 2005).

En base a lo anteriormente mencionado, planteamos una investigación cualitativa, pues con ella se pretende describir como varían los fenómenos en las diferentes circunstancias y cuales son las causas. Se expone como las personas dan sentido a su entorno social y su interpretación. El foco de la investigación cualitativa se centra en la búsqueda de explicaciones subyacentes, percepciones, sentimientos y opiniones de los sujetos de estudio; se pretende conocer la realidad del objeto de la investigación desde el punto de vista de los propios sujetos de estudio. Todas las perspectivas son valiosas, no se busca "la verdad", sino una comprensión detallada de las perspectivas de las personas que participan en el estudio y, para ello, el investigador debe apartar sus propias creencias, perspectivas y predisposiciones. Además, la investigación cualitativa es inductiva, es decir, se desarrollan los conceptos y comprensiones partiendo de los datos que se obtienen en la investigación (Salamanca, 2006).

La investigación sigue una orientación metodológica basada en la Teoría Fundamentada, la cual va estar enraizada en el interaccionismo simbólico (Denzin, 1992; Blummer, 1982). Este ha sido utilizado con éxito en el campo de la Enfermería al tratarse de "una teoría que tiene como concepto central el significado, es decir, las acciones individuales y colectivas son construidas a partir de la interacción entre las personas; y tales acciones dependen del contexto social al que pertenecen" (Ibarra, 2008). En este sentido, la investigación cualitativa enfatiza en el estudio de los procesos y de los significados interesándose por fenómenos y experiencias humanas (De la Cuesta, 1997).

En el caso de nuestro estudio, en base a la orientación filosófica seguida, nos basamos en el razonamiento inductivo, prescindiendo de hipótesis y apoyándonos en un marco teórico previo consistente (De la Cuesta, 2006).

Así pues, planteamos los siguientes objetivos: por un lado, conocer, analizar y describir las expectativas, necesidades y percepciones de los Profesionales de Enfermería, en relación a la satisfacción de la salud laboral como factores influyentes en su calidad de vida. Así como valorar y comparar las expectativas, necesidades y percepciones de estos Profesionales de Enfermería.

\section{MATERIAL Y MÉTODO}

\section{Diseño del estudio}

Para alcanzar los objetivos del estudio, se ha elegido la metodología cualitativa por su utilidad en la profundización en fenómenos de gran interés y complejidad. Ésta metodología, nos permite aprender sobre las experiencias y perspectivas de los participantes, explorar sentimientos, necesidades, expectativas e identificar aquellos factores o situaciones que provocan insatisfacción en los profesionales de enfermería.

\section{Ámbito del estudio}

El estudio de corte cualitativo, se realizará en tres Hospitales Universitarios públicos pertenecientes a tres áreas sanitarias: I, VI y VII.

\section{Participantes del estudio}

Los participantes del estudio, serán los profesionales de enfermería generalistas $y$ profesionales de enfermería obstétrico ginecológica (matrona), mediante su participación en grupos de discusión. El universo de la muestra está compuesto por la plantilla de profesionales de enfermería de los hospitales en estudio. El número de grupos de discusión, 


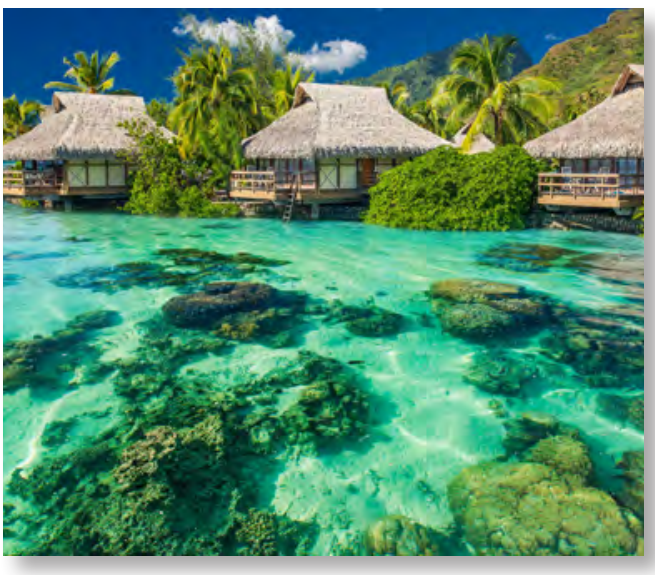

se establecerá según el numero de profesionales de cada hospital, así pues, en el Hospital Clínico Universitario se realizarán dos grupos de discusión con Profesionales de Enfermería generalistas (un grupo del pabellón general y otro del pabellón materno-infantil). En los otros dos Hospitales Universitarios, se realizará un grupo de discusión en cada uno, resultando cuatro grupos. Asimismo, se realizarán dos grupos de discusión con Profesionales de Enfermería especialistas en obstetricia y ginecología en el Hospital Clínico Universitario, ya que es el Hospital de referencia en dicha especialidad para las Áreas Sanitarias I, VI y VII.

La segmentación de los participantes en los grupos de discusión de los profesionales de enfermería generalistas será la siguiente: Dos profesionales de enfermería de unidades de hospitalización quirúrgica, dos de unidades de hospitalización médica, uno de quirófano, uno de urgencias, uno de la unidad de cuidados intensivos y otro de consultas externas. A su vez la de los participantes en los grupos de discusión de los profesionales de enfermería especialistas en obstetricia y ginecología será la siguiente: Dos profesionales de enfermería especialistas en obstetricia y ginecología de consultas externas, dos de la unidad de gestantes y cuatro de la unidad de urgencias y pa- ritorio. Resultando un total de seis grupos de discusión.

Los criterios para la composición de los grupos de discusión respecto a la antigüedad en el puesto, son los siguientes: se tendrán en cuenta los siguientes rangos de edad (1-5 años, 6-10 años, >10 años). En relación a la antigüedad en la profesión los rangos de edad serán (<10 años, 10-30 años, > 30 años). De forma que estén representados todos los criterios anteriormente citados.

\section{Técnica de recogida de datos}

Para la consecución de los objetivos se realizarán grupos de discusión, ya que la interacción del grupo proporcionará más riqueza en el discurso. En la selección estratégica de la muestra se buscará la homogeneidad entre grupos, la heterogeneidad intragrupal y la saturación estructural. Los participantes serán invitados a participar mediante una carta donde se reflejará el objetivo de nuestro estudio, la fecha y lugar de celebración. Posteriormente se confirmará la asistencia por teléfono.

Se realizará la reunión en un lugar neutro, evitando lugares que inhiban o afecten negativamente a la expresión de los grupos. Se realizará la grabación magnetofónica completa del grupo para su posterior transcripción. Las reuniones serán moderadas por un miembro del equipo investigador y se recogerán notas de campo por parte de un segundo observador. Después de la celebración de cada grupo, el moderador y el observador harán una primera audición provisional.

Asimismo, para contribuir a la consecución de los objetivos, se realizará una observación sistemática en los hospitales objeto de estudio que explore: ¿Cómo se relacionan los profesionales? ¿Cómo organizan sus actividades? ¿Están motivados en cuanto a la presta- 
ción de cuidados? ¿Qué expectativas y necesidades laborales tienen? ¿Cómo se sienten? Así como la utilización y registro de los diarios de campo de los investigadores.

Las preguntas guía se basarán en las siguientes cuestiones: condiciones del entorno laboral, flexibilidad del método de trabajo, relación con los compañeros, reconocimiento laboral, relaciones con los superiores, responsabilidad asignada, uso de capacidades personales, promoción laboral, horarios, estabilidad en el empleo, oportunidades de formación, satisfacción en líneas generales, etc.

\section{Periodo de estudio}

El estudio se realizará durante los años 2013 a 2015.

\section{Análisis de la información}

Se realizará un análisis preliminar después de cada grupo de discusión. Resumen sobre hallazgos, interpretaciones, observaciones acerca de la dinámica de la reunión, que nos servirán para buscar esquemas de codificación que sirvan, no solo para estructurar el contenido de todas la entrevistas sino también la necesidad de variar el número y la estructura de otros participantes con el fin de alcanzar la saturación de los datos.

Durante el análisis de los mismos, se realizará una contra-codificación entre el equipo investigador, así como una doble verificación de las relaciones entre los códigos y el modelo explicativo, con el fin de intentar ofrecer los resultados más fiables. Se realizarán comparaciones constantes de la codificación, categorización, subcategorización y relación entre categorías con la finalidad de elaborar explicaciones que den sentido a los textos. El proceso de categorización del análisis se perfilará con ayuda de informes analíticos de bibliografía previa. Se utilizará el programa informático para datos cualitativos Atlas-ti

\section{Aspectos éticos}

Se cumplirá escrupulosamente y en todo momento con el compromiso del cumplimiento de las normas éticas de investigación y de los requerimientos legales imprescindibles para realizar este estudio. De igual forma se respetará en todo momento la Ley Orgánica 15/1999, de 13 de diciembre, de Protección de Datos de Carácter Personal, así como las autorizaciones a los Gerentes y comités de ética de las Áreas de Salud estudiadas para la realización de los Grupos de Discusión a los profesionales.

Asimismo, a todos los participantes se les citará personalmente para explicarles brevemente el objeto del estudio, su técnica y porqué han sido invitados a participar. Se les dará la información que precisen y se les pedirá que firmen el formulario de Consentimiento Informado. De manera particular se llevará especial atención en el cumplimiento de los siguientes aspectos éticos: Voluntariedad a la hora de participar en la investigación. Anonimato de las personas encuestadas. Confidencialidad de los datos obtenidos en el estudio. Almacenaje y mantenimiento de los datos recogidos en lugar seguro impidiendo que ninguna persona que no pertenezca al equipo investigador pueda hacer uso de los mismos. Inocuidad de los resultados del estudio para ninguno de los participantes en la investigación.

\section{Limitaciones del estudio}

Las personas que acepten participar en el estudio, seguramente serán las más colaboradoras. Puede que la utilización de una grabadora y la técnica de observación participante ejerzan una presión que pueda disminuir o bloquear la espontaneidad en algunos participantes y limitar así la información que apor- 
ten. El hecho de realizar la investigación en una única Comunidad Autónoma y en tres hospitales, puede disminuir la posibilidad de generalización de los resultados, pero ese no es el objetivo de la investigación cualitativa, sino el del conocimiento en profundidad de un fenómeno. El muestreo teórico puede producir una falta de captación de toda la problemática del fenómeno en la medida en la que lo haría el muestreo de máxima variedad, sin embargo, por cuestiones de factibilidad y teniendo en cuenta que se buscará la saturación de los datos consideramos adecuada la técnica.

\section{RESULTADOS}

A continuación, se describen los resultados del estudio cuantitativo de la satisfacción laboral realizado con profesionales sanitarios de uno de los Hospital Universitario en estudio, estos resultados nos han servido de base a la hora de plantear el presente proyecto de investigación, dado que el personal de enfermería fue el que mostró una mayor insatisfacción laboral (Carrillo, 2011). El instrumento de medición que se utilizó en dicha investigación cualitativa fue la Escala General de Satisfacción (Overall Job Satisfacción) (War, Cook and Wall, 1979) y adaptada y validada al castellano (Pérez y Fidalgo, 1995). Esta escala solicita a los trabajadores el nivel de satisfacción con distintos elementos de la vida laboral, con un sistema de respuesta tipo Likert de siete puntos de anclaje, desde 1 "muy insatisfecho" hasta 7 "muy satisfecho". Los 15 ítems de la escala original están divididos en dos subescalas, una de factores intrínsecos, referentes al propio contenido del trabajo formada por siete ítems. Y otra de factores extrínsecos, vinculados con el contexto donde se desarrolla la actividad laboral que la constituyen ocho ítems. Esta escala de satisfacción laboral, es ampliamente usada y se encuentra insertada dentro del marco conceptual de la Teoría Bifactorial de Herzberg.

Para un universo objeto de estudio de 1194 profesionales sanitarios y teniendo presente que la muestra final fue de 546 encuestados, el porcentaje de participación fue del $45.81 \%$.

Los tres aspectos mejor valorados por los profesionales, son los que hacen referencia tanto a los compañeros de trabajo, al superior inmediato y al horario de trabajo con unas valoraciones medias de 5.9, 5.7 y 5.3 respectivamente.

Los siguientes aspectos mejor valorados todos ellos con una valoración media superior a 5, son los relativos a la responsabilidad asignada, estabilidad en el empleo y la variedad en las tareas, con unas valoraciones medias de 5.2, 5.2 y 5.1 respectivamente.

Los resultados de la encuesta ponen de manifiesto que, la mayor parte de los aspectos en análisis obtienen una valoración media del nivel de satisfacción. Así, hasta 8 de los 16 ítems en análisis son calificados con una valoración de entre 4 y 5 .

Finalmente, los dos únicos aspectos que obtienen niveles medios de satisfacción negativos, es decir, inferiores al 4 (de la escala Likert considerada, de 1 a 7), son las "Relaciones entre dirección y trabajadores del Hospital” y "El modo en el que el Hospital está gestionado". Ambos factores son los que aportan una menor satisfacción laboral, tal y como puede comprobarse en los gráficos que siguen. Con una valoración media ambos de 3.8 y 3.6 respectivamente.

La última pregunta del cuestionario solicita a los entrevistados que valoren, en líneas generales, y considerando globalmente todos los aspectos anteriores, el "nivel de satisfacción con el trabajo desempeñado en el Hospital”. El nivel de satisfacción general medio expresado 
por los entrevistados con el trabajo desempeñado se sitúa en el 5.4 en la escala de 1 a 7.

Las diferencias observadas en los distintos niveles de satisfacción atendiendo a la categoría profesional, sugieren que, para la mayor parte de los aspectos en análisis, el grupo de Gerencia y Dirección presentan los niveles más elevados de satisfacción, seguido por el de los Médicos Residentes. Frente a estos grupos, el de Enfermería destacaría por ser el que ofrece niveles de satisfacción más reducidos.

\section{DISCUSIÓN Y CONCLUSIONES}

Con este tipo de investigación se podrán analizar y describir las expectativas, necesidades, valores y percepciones de los profesionales de enfermería respecto a su satisfacción de la salud laboral a través de grupos de discusión, siendo un medio de expresión de las ideologías sociales para captar las representaciones de la cultura o fenómeno que estamos investigando. Consideramos apropiado el uso de esta técnica por las ventajas inherentes de flexibilidad, alta validez, resultados rápidos y bajo coste (Guardián, 2007), por ser muy rica en producción de información y tener la capacidad de capturar material de la vida real en ambientes sociales. Al tratarse de una herramienta cuidadosamente planificada donde enfocamos el lenguaje como expresión (Ortí, 1986), extraemos los discursos que nos aportarán las representaciones ideológicas de los profesionales de enfermería generalistas y especialistas objeto de nuestra investigación. A través de las autoconfesiones de los participantes (Kruege, 1991), pretendemos profundizar en las expectativas, conocimientos, opiniones e ideologías, así como los sentimientos y emociones culturalmente construidos (Téllez, 2007), expresados por aquellos que tienen que ver o que están directamente involucrados en la realidad social que se pretende investigar.

El diseño del grupo se conformará con criterios de homogeneidad controlada con la finalidad de revisar los campos sociales y simbólicos, pues en ellos se construyen los sentidos compartidos, los lugares comunes y los sistemas tópicos. La excesiva lejanía entre los miembros, provocaría conflictividad potencial de los mismos, obstaculizando la elaboración y negociación de los sentidos atribuidos a las representaciones y categorías sociales (Bourdieu, 1989).

Por otro lado, dado que la investigación está enmarcada en la Teoría Fundamentada y ésta, a su vez, enraizada en el interaccionismo simbólico, el grupo de discusión supone un instrumento de recogida de datos eficaz para captar los universos simbólicos a través de los cuales se interpreta la realidad social en la que están involucrados los sujetos y les dota de significado (Berger y Luckman, 1979). Así mismo, el lenguaje hablado indaga en los procesos de asignación de símbolos con significado y nos hace comprender los procesos de asignación de los significados (Baez, 2009), por tanto esta dinámica grupal aportaría datos acerca de la naturaleza de la interacción que se establece entre los profesionales de enfermería en el escenario laboral en el que se encuentran inmersos.

El proyecto de investigación comenzó a realizarse en febrero de 2013 y hasta ahora todo transcurre dentro de los plazos establecidos y sin incidencias. Tras haber realizado la búsqueda bibliográfica pertinente para situarnos en el estado de la cuestión de nuestro proyecto, hemos construido la herramienta que nos sirve como guía para los grupos de discusión. Una vez establecidos los campos a indagar, las preguntas clave se han validado mediante entrevista individual, mediante la cual se ha po- 
dido reestructurar la guía y añadir campos de los que nos interesa su investigación. Cuando teníamos la herramienta diseñada y validada hemos solicitado los permisos necesarios en las Áreas de Salud donde dirigimos nuestro estudio. La respuesta ha sido inmediata y satisfactoria, por lo que nos pusimos a construir tres grupos de discusión, uno de enfermeros generalistas del Área VI (noviembre de 2013) y dos de matronas: uno del Hospital Clínico Universitario (Área I ) y otro de Primaria de las Áreas I, VI y VII.

Hasta el momento hemos conseguido buena respuesta por parte de los participantes en cuanto a asistencia como a participación. Por nuestra parte, la organización logística ha sido pertinente y los que han trabajado como entrevistadores han dirigido las conversaciones de forma correcta, tanto en el manejo del grupo como en el control de tiempos.

Actualmente nos encontramos en fase de transcripción de los datos que hasta ahora hemos recogido.

\section{BIBLIOGRÁFIA}

- Báez y Pérez de Tudela, J. (2009) Investigación Cualitativa (2 ed.). ESIC Editorial, Madrid.

- Berger, P. y Luckman T. (1979) La construcción de la realidad. Amorrortu Editores, Buenos Aires.

- Blummer, H. (1982) El interaccionismo simbólico: Perspectiva y método. Hora, Barcelona.

- Bourdieu, P. (1989) La ilusión biográfica. Historia y Fuente Oral 2: 27-33. Available http://es.scribd.com/ doc/62808818/Bourdieu-Pierre-1989-la-ilusión-biografica-en-Historia-y-fuente-oralno-2.

- Carrillo, C. (2011) Un estudio sobre la satisfacción de la vida laboral en profesionales sanitarios del Hospital General Universitario José María Morales Meseguer. Región de Murcia. Tesis Doctoral. Murcia, Universidad de Murcia.

- De la Cuesta, C. (1997). Características de la investiga- ción cualitativa y su relación con la enfermería. Investigación y Educación en Enfermería 15 (2): 14-24. Available http://rua.ua.es/dspace/bitstream/10045/18349/1/ De\%20la\%20Cuesta_Caracteristicas\%20de\%20la\%20 Investigacion.pdf.

- De la Cuesta, C. (2006) La Teoría Fundamentada como herramienta de análisis. Revista Cultura de los Cuidados 20: 136-140.

- Denzin, N.K. (1992) Symbolic interaccionism and cultural studies: the politics of interpretation. Blackwell, Cambridge.

- Flores, N; Jenaro, C; González-Gil, F; Robaina, F; Martín, M.V. (2010) Quality of working life in healthcare services. En: Ciurea, A.V; Cooper, C.L; Avram, E. ed. Management in health organizations, The 21st century perspectives, Bucarest: 129-162.

- García de Diego, J.M; Ranchal Romero, J; Biedma Velázquez, L; Serrano del Rosal, A. (2008) Preferencias y expectativas de los médicos residentes en Andalucía. Instituto de Estudios Sociales Avanzados, Córdoba.

- Guardián, A. (2007) El paradigma cualitativo en la investigación socio-educativa. Colección IDER, Costa Rica.

- Ibarra, T.X. (2008) El interaccionismo Simbólico y los Cuidados de Enfermos Crónicos en el Ámbito Comunitario. Revista Cultura de los Cuidados 12 (24): 94-106.

- Kruege, R. (1991) El grupo de discusión. Pirámide, Madrid.

- Organización Mundial de la Salud. (2013) Salud Ocupacional. Available http://www.who.int/topics/occupational_health/es/

- Ortí, A. (1986) La apertura y el enfoque cualitativo o estructural: la entrevista abierta semidirectiva y la discusión en grupo. En García, M.; Ibáñez, J.; Alvira, F. ed. El análisis de la realidad social, Alianza Universidad, Madrid: 153-185.

- Peiró, J.M. (1986) Psicología de la organización. (2a ed.) UNED, Madrid.

- Peiró, J.M.; Silla Guerola I.A.; Sanz Cuesta, T.; Rodríguez Escobar, J.; García Más, J.L. (2004) Satisfacción laboral de los profesionales de atención primaria. Psiquis: Revista de Psiquiatría, Psicología Médica y Psicosomática 25 (4): 129-140. 


\section{Cultura de las Cuidados}

- Pérez, J.; Fidalgo, M. (1995) NTP: 394: Satisfacción laboral: escala general de satisfacción. (Informe NTP: 394). Available http://www.insht.es/InshtWeb/ Contenidos/Documentacion/FichasTecnicas/NTP/ Ficheros/301a400/ntp_394.pdf

- Robles García, M.; Dierssen Sotos, T.; Martínez Ochoa, E.; Herrera Carral, P.; Díaz Mendi, A.; Llorca Díaz, J. (2005) Variables relacionadas con la satisfacción laboral: un estudio transversal a partir del modelo EFQM. Gaceta Sanitaria 19 (2): 127-134.

- Sáez, M.C. (1999) Motivación en el trabajo. En: García, M. ed. Psicología del Trabajo y de las Organizaciones. Fundamentos del Comportamiento en las Organizaciones, Diego Marín, Murcia: 107-130.

- Sáez, M.C. (1999). Satisfacción Laboral. En: García, M. ed. Psicología del Trabajo y de las Organizaciones. Fundamentos del Comportamiento en las Organizaciones, Diego Marín, Murcia: 131-146.

- Salamanca, A.B. (2006) La investigación cualitativa en ciencias de la salud. Revista Nure Investigación 24. Available http://www.fuden.es/FICHEROS_ADMINISTRADOR/F_METODOLOGICA/ FMeTd_24.pdf

- Téllez, A. (2007) La investigación antropológica. Editorial Club Universitario, Alicante.

- Warr, T.; Cook, P.; Wall, K. (1979) Scales for the measurement of some work attitudes and aspects of psychological well-being. Journal of OccupationalPsychology 52: 129-148.

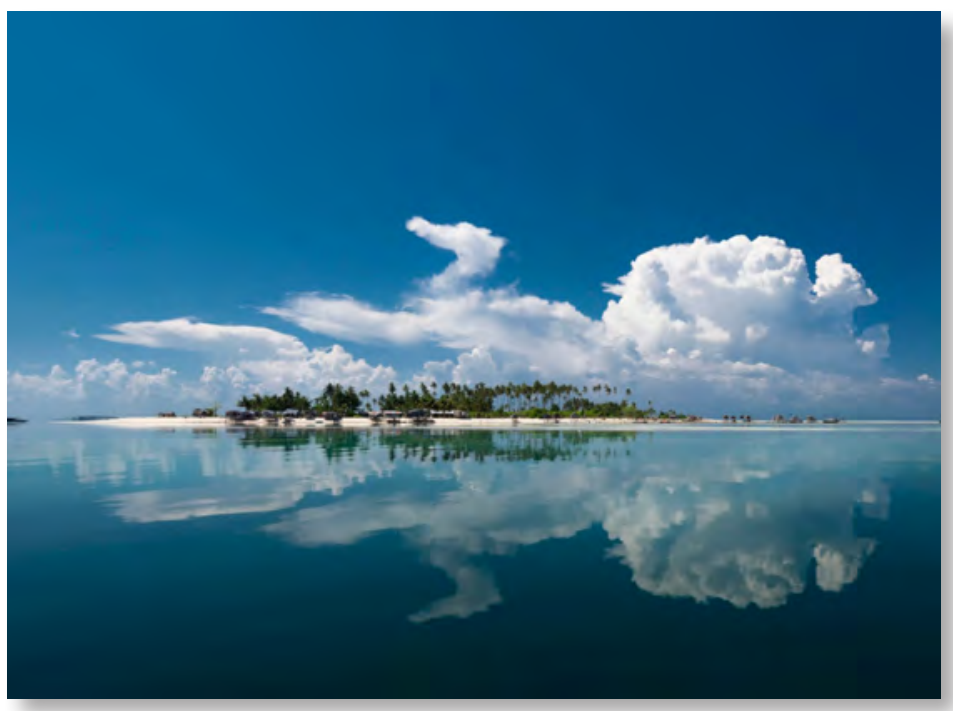

